University of Wollongong

Research Online

Faculty of Engineering and Information

Faculty of Engineering and Information

Sciences - Papers: Part B

Sciences

2018

Development of ciprofloxacin-loaded poly(vinyl alcohol) dry powder formulations for lung delivery

Dina M. Morais da Silva

University of Wollongong

Roberto Paleco

University of Sydney

Daniela Traini

University of Sydney

Vitor Sencadas

University of Wollongong, victors@uow.edu.au

Follow this and additional works at: https://ro.uow.edu.au/eispapers1

Part of the Engineering Commons, and the Science and Technology Studies Commons

Research Online is the open access institutional repository for the University of Wollongong. For further information contact the UOW Library: research-pubs@uow.edu.au 


\title{
Development of ciprofloxacin-loaded poly(vinyl alcohol) dry powder formulations for lung delivery
}

\author{
Abstract \\ Polymeric microparticles are micro carriers for the sustained drug delivery of drugs in the lungs, used as \\ alternatives to the use of established excipients. This study aims to develop and characterize inhalable \\ ciprofloxacin (CPx)-loaded poly(vinyl alcohol) (PVA) microparticles by a single-step spray-drying \\ procedure. \\ Disciplines \\ Engineering | Science and Technology Studies

\section{Publication Details} \\ Silva, D. M., Paleco, R., Traini, D. \& Sencadas, V. (2018). Development of ciprofloxacin-loaded poly(vinyl \\ alcohol) dry powder formulations for lung delivery. International Journal of Pharmaceutics, 547 (1-2), \\ 114-121.
}


Development of ciprofloxacin-loaded poly(vinyl alcohol) dry powder formulations for lung delivery

Dina M. Silva ${ }^{a^{*}}$, Roberto Paleco $^{b}$, Daniela Traini ${ }^{b}$, Vitor Sencadas $^{a, c^{*}}$

${ }^{a}$ School of Mechanical, Materials, Mechatronic and Biomedical Engineering, University of Wollongong, Wollongong, NSW 2522, Australia

${ }^{\mathrm{b}}$ Respiratory Technology, Woolcock Institute of Medical Research and Discipline of Pharmacology, Sydney Medical School, The University of Sydney, Sydney, Australia

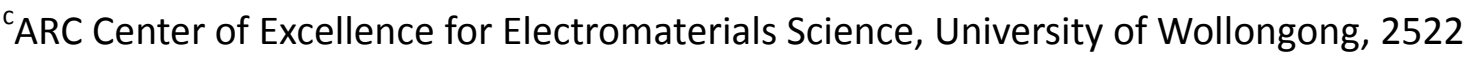
NSW, Australia

${ }^{*}$ Corresponding authors:

Vitor Sencadas: victors@uow.edu.au; vsencadas@gmail.com

Dina M. Silva: dsilva@uow.edu.au; dina.morais.silva@gmail.com

School of Mechanical, Materials, Mechatronic and Biomedical Engineering, University of Wollongong, Northfields Ave, Wollongong, NSW 2522, Australia Ph: +61242214614

Keywords: Poly(vinyl alcohol), drug delivery, spray-drying, lung administration, next generation impactor. 


\section{Abstract:}

Polymeric microparticles are micro carriers for the sustained drug delivery of drugs in the lungs, used as alternatives to the use of established excipients. This study aims to develop and characterize inhalable ciprofloxacin (CPx)-loaded poly(vinyl alcohol) (PVA) microparticles by a single-step spray-drying procedure. The optimization of the processing parameters was achieved by an orthogonal design of the most relevant processing parameters (polymer concentration, feed rate and inlet temperature). The obtained spray-dried particles showed a drug encapsulation efficiency higher than 90\%. Furthermore, PVA-CPx formulations, with drug contents up to $10 \mathrm{wt} . \%$, showed a morphology and size suitable for inhalation, with a sustained release profile over $24 \mathrm{~h}$. Data from Fourier transformed infra-red spectroscopy and differential scanning calorimetry indicated absence of interaction between the polymer matrix and the drug. Aerodynamic assessment of PVA-CPx 10 wt.\% was determined by the next generation impactor (NGI), using spray-dried CPx as a control. The results showed improved values of mass median aerodynamic diameter $(5.06 \pm 0.10 \mu \mathrm{m})$ and a fine particle fraction $(39.78 \pm 0.98 \%)$ when comparing with the CPx alone $(5.33 \pm$ $0.39 \mu \mathrm{m}$ and $30.43 \pm 1.38 \%)$. This study highlights the potential of spray-dried PVA microparticles as drug carriers for lung local delivery of antibiotics.

\section{Abbreviations}

PVA - Poly(vinyl alcohol); CPx - Ciprofloxacin; SD - spray-dried; NGI - next generation impactor; MMAD - mass median aerodynamic diameter; FPF - fine particle fraction; DPI - dry powder inhaler. 


\section{Introduction}

The administration of pharmaceuticals via the pulmonary route has been a widely used therapy to address pulmonary diseases such as asthma, cystic fibrosis, and chronic infections. Lungs are an attractive delivery route for systemic therapies due to the highly vasculated, large surface area $\left(100 \mathrm{~m}^{2}\right)$, thin alveolar epithelium (0.1-0.2 $\left.\mu \mathrm{m}\right)$, and low metabolic activity, which accounts for improved absorption and bioavailability, when compared with intravenous and oral administration of drugs (Farr and Otulana, 2006; Manca et al., 2015; Patton, 1996).

Regardless the final target site, the same key requirements must be accomplished to have an effective therapeutic effect, which basically rely on device design and powder formulation. Apart from the device performance itself, the powder formulation properties such as particle size, flow, shape, surface properties and drug-carrier interaction, strongly influences the therapeutic dose (Atkins, 2005). The deposition site is mainly driven by the particle mass median aerodynamic diameter (MMAD) and this relationship establishes the size range between 1 and $5 \mu \mathrm{m}$ as the peak of particle fraction that reaches the small airways and alveolar region (Labiris and Dolovich, 2003; Patton and Byron, 2007), which increases the residence time in the lungs due to a slower mucociliary clearance (Sakagami and Byron, 2005). Up to date, poly(lactic-coglycolic acid) (PLGA) is the most studied polymer for inhalable therapeutics owing to its biocompatibility and biodegradability (Dailey et al., 2006; Hara et al., 2008). Ungaro et al. (Ungaro et al., 2012) developed PLGA-based nanoparticles for antibiotic encapsulation, using hydrophilic helper polymers to modulate the release rate and the lung deposition in a multistep processing. They added lactose as a microcarrier to promote an efficient aerosolization of the nanoparticles, and showed an MMAD of 
$3.4 \pm 1.7$ and $5.7 \pm 1.8 \mu \mathrm{m}$ with a fine particle fraction (FPF) of $52 \pm 1$ and $38 \pm$ $1 \%$, respectively, for the formulations with the best performances. Rawat et al. reported that heparin-loaded PLGA microspheres produced by double emulsion techniques were within the inhalable size range, but both the entrapment efficiency (up to 55\%) and half-life (up to 6-fold) were only increased by the addition of chemical additives (Rawat et al., 2008). Manca and co-workers also developed carriers for pulmonary administration of rifampicin based on gelatin (Manca et al., 2013) and polymer- coated liposomes (Manca et al., 2014), which displayed good aerodynamic properties and an efficient drug internalization by A540 cells.

The in vivo effects of inhalable nanoparticles from alginate (Zahoor et al., 2005), albumin (Woods et al., 2015) and chitosan (Trapani et al., 2013) also showed promising results when compared with oral administration, although natural polymers often raise some issues due to the presence of endotoxins (Pilcer and Amighi, 2010; Sivadas et al., 2008).

There is a constant demand to use hydrophilic polymers, that can be used as vehicles to carry and release the therapeutic agents in a controlled fashion. Poly(vinyl alcohol) (PVA) is a synthetic water soluble polymer, completely biodegradable and its microspheres as drug carriers have already been approved by Food and Drug Administration (FDA) for embolization (Chaouat et al., 2008). PVA microspheres can be prepared by electrospray (Felice et al., 2015), but the processing method is unstable over time, with low yields and not scalable for an industrial application. On the other hand, spray drying offer a unique opportunity to tune and optimize the processing parameters from bench scale to large scale production. 
The orthogonal factorial design is a mathematical method to analyze a multifactorial and multilevel process, which uses only a fraction of the experiments needed for a full factorial design, still obtaining accurate estimates (Lee et al., 2013; Zheng et al., 2014). This method allows to determine the relative importance of each factor and provides the level optimization for each tested factor (Xie et al., 2015). Several works explored this approach for the optimization parameters of polysaccharides extraction (RenJie, 2008) and electrospinning fibers (Vild et al., 2016; Xie et al., 2015), for the sensitivity analysis of biometric systems (Lee et al., 2013) and optimization of laser-induced breakdown spectroscopy in aluminum alloys (Zheng et al., 2014). The use of a design of experiments (DOE) approach, helps to determine which factors have more influence in the process, saving experimental time and raw materials to establish the optimum conditions.

In this work, the spray-drying processing of inhalable polymeric particles was optimized through an orthogonal design by the systematic manipulation of processing parameters, using PVA (polyvinyl alcohol), a synthetic biocompatible and biodegradable polymer, as a carrier.

Antibiotic therapy is often limited by systemic toxicity raised by the high doses administrated by oral or parenteral formulations (Rybak et al., 1999). Hence, locally and controlled delivery of antibiotics offers a potential alternative to overcome those drawbacks. Ciprofloxacin (CPx), a broad-spectrum fluoroquinolone used to treat respiratory infections by oral and parenteral formulations, has been used as a model drug in this study.

Accordingly, formulations of PVA-CPx with CPx contents up to 10 wt.\% were prepared using the optimal conditions obtained from the orthogonal design. The developed 
formulations were characterized in terms of morphology, size distribution, physicochemical and thermal properties, and in vitro release kinetics. The aerodynamic performance of this formulation was also investigated in vitro using the next generation impactor.

\section{Material and methods}

\subsection{Materials}

Poly(vinyl alcohol) (PVA; $M_{W} 85-124$ kDa; +99 \% hydrolyzed), ciprofloxacin (CPx; purity $\geq 98 \%$ ), sodium chloride $(\mathrm{NaCl})$, potassium chloride $(\mathrm{KCl})$, sodium phosphate dibasic $\left(\mathrm{Na}_{2} \mathrm{HPO}_{4}\right)$, potassium phosphate monobasic $\left(\mathrm{KH}_{2} \mathrm{PO}_{4}\right)$ and hydrochloric acid $(\mathrm{HCl})$ were purchased from Sigma-Aldrich (Australia), and used as received. Deionized water was produced by an Elix Essential 3 (Millipore) water purification system.

\subsection{Microparticles processing}

PVA and PVA-CPx microparticles were obtained with an in-house built spray drying system, using PVA aqueous solutions as feed stock. For PVA-CPx formulations, a stock solution of $\mathrm{CPx}$ in $0.1 \mathrm{M} \mathrm{HCl}$ was added to the PVA solution to a final CPx concentration of 2,5 , and $10 \mathrm{wt} . \%$. Initially, the spray drying parameters were optimized for the drug free PVA microparticles by an orthogonal design table $L_{9}$, using Orthogonal Design Assistant II software (Sharetop Software Studio). The experimental parameters of feed concentration, feed rate and inlet temperature are described in Table 1. 


\subsection{Rheological analysis}

The rheological behavior of feed stock solutions with different PVA concentrations $(0.5,1$ and $2 \% \mathrm{w} / \mathrm{v})$ was evaluated using a parallel plate $(\varnothing=20 \mathrm{~mm})$ geometry rheometer (MCR 301, Anton Paar) with a $0.3 \mathrm{~mm}$ gap to ensure adequate filling of the samples over the testing plate. Viscosity was measured as a function of shear rate within the range of $1-1000 \mathrm{~s}^{1}$, at $25 \stackrel{\circ}{ } \mathrm{C}$.

\subsection{Physico-chemical characterization}

\subsubsection{Scanning electron microscopy (SEM)}

The dry particles were coated with a $15 \mathrm{~nm}$ gold layer using a sputter coater (Smart Coater, JEOL) and their morphology analyzed by scanning electron microscopy (JCM6000PLUS Neoscope, JEOL), with an accelerating voltage of $15 \mathrm{kV}$. Particles average diameter and their distribution was calculated over 300 particles from SEM microphotographs (1,000 x magnification) using the Image J software (Schneider et al., 2012). Particles dispersity was quantitatively evaluated by the coefficient of variation $\left(C_{V}\right)$, defined as $C_{V}=\sigma / \mu$, where $\sigma$ represents the standard deviation of the mean particle size $(\mu)$ (Felice et al., 2015).

\subsubsection{Fourier Transform Infrared Spectroscopy (FTIR)}

FTIR measurements were performed on dry powders using a Shimadzu IRAffinity-1S apparatus in ATR (attenuated total reflectance) mode from 4000 to $600 \mathrm{~cm}^{-1}$, at room temperature. FTIR spectra were collected after 24 scans with a resolution of $1 \mathrm{~cm}^{-1}$. Background spectra were subtracted from the sample ones. 


\subsubsection{Raman Analysis}

Raman spectra was performed on a Horiba Jobin Yvon LabRAM HR 800 Raman spectrometer, using excitation of $\mathrm{He}-\mathrm{Ne} 632.8 \mathrm{~nm}$ with a power of $13.5 \mathrm{~mW}$, and a spectral resolution of $2 \mathrm{~cm}^{-1}$.

\subsubsection{Differential scanning calorimetry (DSC)}

Differential scanning calorimetry measurements were performed with a Polyma 214 apparatus (Netzsch). Samples weighting around $5 \mathrm{mg}$ were placed inside $40 \mu \mathrm{L}$ aluminum pans with pierced lids, heated between 30 and $250^{\circ} \mathrm{C}$ at a rate of $10^{\circ} \mathrm{C} / \mathrm{min}$. All experiments were performed under a nitrogen purge.

\subsubsection{Thermogravimetrical analysis (TGA)}

Thermogravimetrical analysis (TGA) was conducted in a TGA 4000 system (PerkinElmer) using a heating rate scan of $20 \stackrel{\circ}{ } \mathrm{C} \mathrm{min}^{-1}$, under a nitrogen atmosphere.

\subsubsection{Particle size distribution analysis}

The size distribution of the PVA and PVA-CPx 10 wt.\% microparticles were determined by laser diffraction using a Malvern Mastersizer 3000 (Malvern Instruments Ltd.) with a Scirocco dry powder feeding cell. Samples ( $\approx 10 \mathrm{mg})$, analyzed in triplicates, were dispersed in air using a feed pressure of 4 bar with an obscuration between $0.1 \%$ and $10 \%$, and a refractive index of 1.52 .

2.4.7 Dynamic vapor sorption (DVS) 
The moisture sorption characteristics of PVA and PVA-CPx 10 wt.\% microparticles were assessed by dynamic vapor sorption (DVS-1 Intrinsic, Surface Measurement Systems Ltd.). Samples (10-30 $\mathrm{mg}$ ) were dried at $0 \%$ relative humidity $(\mathrm{RH})$ at $25 \% \mathrm{C}$ before exposure to two sorption cycles from 0 to $90 \%$, at $10 \%$ increments. At each stage, the moisture sorption equilibrium was determined by a change in mass-to-time ratio $(d m / d t)$ of $0.0005 \% \cdot \min ^{-1}$.

\subsection{Ciprofloxacin content}

The $\mathrm{CPx}$ content was determined by re-suspending the microparticles in $0.1 \mathrm{M} \mathrm{HCl}$, in ultrasonic bath ( $250 \mathrm{HD}$, Soniclean) for $6 \mathrm{~h}$. The suspensions were centrifuged $(10,000$ $\mathrm{rpm}, 10 \mathrm{~min}$ ) to remove the polymeric debris. CPx concentration was determined spectrophotometer (UV1800, Shimadzu) at a wavelength of $277 \mathrm{~nm}$, using a calibration curve of $\mathrm{CPx}$ in $\mathrm{HCl}(0.1 \mathrm{M})$. Calibration standards were linear in the range of $0.5-15 \mu \mathrm{g} / \mathrm{mL}\left(R^{2}=0.999\right)$. The drug content and entrapment efficiency were calculated with Equation 1 and 2:

$$
\text { CPx content }(\%)=\frac{\text { amount of CPx in microparticles }}{\text { amount of microparticles taken }} \times 100
$$

$$
\text { Entrapment efficiency }(\%)=\frac{C P x \text { content }}{\text { Theoretical content }} \times 100
$$

\subsection{In vitro drug release}

An accurately weighed quantity of PVA-CPx formulations were transferred to a dialysis membrane with a nominal cutoff of 1 kDa (Cellu.Sep H1, Fisher Biotec) keeping 
constant the CPx amount $(914 \mu \mathrm{g})$. The membranes were then immersed in $200 \mathrm{~mL}$ of PBS ( $\mathrm{pH} 7.4)$, under magnetic stirring at 37 ㅇ․ At predetermined time intervals up to $24 \mathrm{~h}, 2 \mathrm{~mL}$ of the release medium was withdrawn and replaced with fresh PBS. The release experiments were designed to ensure sink conditions for CPx following a previous work (Adi et al., 2010). The aqueous solubility of ciprofloxacin has been reported to be $30 \mathrm{mg} / \mathrm{mL}$ at $20 \circ \mathrm{C}$ (Varanda et al., 2006), hence CPx concentration in our experiments $(4.57 \mu \mathrm{g} / \mathrm{mL})$ would be 6500 times less than the solubility limit. The release medium was measured at $270 \mathrm{~nm}$ using a spectrophotometer (UV1800, Shimadzu) and the CPx concentration was calculated using a calibration curve of CPx in PBS, with results expressed as \% cumulative release relative to the initial weight of CPX loaded in the particles. Analysis was performed in triplicates and results were averaged.

The experimental results were fitted using the Korsmeyer-Peppas model (Equation 3) to further evaluate the release kinetics.

$\frac{M_{t}}{M_{\infty}}=k t^{n}$

where $M_{t} / M_{\infty}$ is a fraction of drug released at time $t, k$ is the rate constant, and $n$ is the exponent that characterizes the release mechanism (Ritger and Peppas, 1987b; Siepmann and Peppas, 2001).

\subsection{In vitro aerodynamic performance}

The aerodynamic properties of PVA and PVA-CPx 10 wt.\% microparticles was evaluated using a next generation cascade impactor (NGI; Westech UK) equipped with an 
induction port, as per United States Pharmacopeia (USP) methodology. The flow rate through the NGI was adjusted to $60 \pm 2$ L. $\mathrm{min}^{-1}$ using a pump and calibrated flow meter (TSI 4040; TSI Instruments Ltd., USA). The PVA and PVA-CPx microparticles were loaded into size 3 gelatin capsules (Capsugel, Australia) and placed in the dosage chamber of a high-resistance dry powder device (DPI, RSO1, Plastiape, Italy). The inhaler was connected to a 3D printed mouthpiece adaptor, that was inserted into the USP induction port, and the microparticles were dispersed over a $4 \mathrm{~s}$ period to simulate one breath. Following actuation, the device, capsule, mouthpiece, throat, and all the stages of the NGI were washed with MilliQ water. Ciprofloxacin was extracted in water suspensions at $95{ }^{\circ} \mathrm{C}$ for $1 \mathrm{~h}$, sonicated for $30 \mathrm{~min}$ and filtered with a $0.45 \mu \mathrm{m}$ nylon filter, prior to quantification. Each sample was tested in triplicate. Mass median aerodynamic diameter (MMAD), geometric standard deviation (GSD) and fine particle fraction (FPF) (mass of emitted dose with aerodynamic diameter $\leq 5 \mu \mathrm{m}$ ) were calculated from the NGI results.

\subsection{Data analysis}

Quantitative data are presented as mean \pm standard deviation (SD). Significant differences were determined by one-way ANOVA analysis with Tukey`s HSD post hoc test or unpaired 2-tailed t-tests (for comparisons between two groups) using GraphPad Prism Software (v 6.01). Statistical differences were considered when $p<0.05$. 


\section{Results and Discussion}

\subsection{Particle processing}

There is a demand for alternative excipients for pulmonary drug delivery and, specifically for polymeric approaches which may assist in the aerosolization process while promoting a more sustained release over time. However, there is yet limited information relating the processing and performance of polymeric inhalable systems, and those already available often involve complex multistep processing and /or the need for an extra carrier or incorporation of additives to improve drug loading efficiency (Rawat et al., 2008; Ungaro et al., 2012).

This work reports the development of inhalable polymeric particles for ciprofloxacin lung delivery. A preliminary step was carried out to optimize the spray-drying parameters for PVA alone. Using an orthogonal design (Vild et al., 2016), three factors (feed concentration, feed rate and inlet temperature) were screened among three levels (Table 1) and the effects were evaluated by obtained mean particle diameter (Table S1 and Figure S1 for size distributions). The water content (Figure S2) calculated by the TGA profiles in the region of water evaporation, ranged between 0.3 and $5.5 \%$ for all the experimental runs. The impact of these factors on the particle diameter is depicted in Figure 1a, where the increase of feed concentration appears to induce an increase in particle size, despite the similarity in the feed solution viscosity for the tested concentrations (Figure $1 b$ ). Indeed, the determination of the range values $(R)$ (Table 2 ) attributed to the feed concentration the highest impact on the process, followed by the feed rate and inlet temperature. Accordingly, the optimal levels were settled as levels 1, 3 and 1 for concentration $(0.5 \% \mathrm{w} / \mathrm{v})$, feed rate $\left(308 \mathrm{~mL} \cdot \mathrm{h}^{-1}\right)$ and inlet temperature $(105 \stackrel{\circ}{\circ})$, 
respectively. Despite this, the feed rate used in this experiment was reduced to level 2 $\left(206 \mathrm{~mL} \cdot \mathrm{h}^{-1}\right)$, due to the accumulation of water vapor on the collecting jar, jeopardizing particle integrity and leading to a non-continuous process.

PVA microparticles containing ciprofloxacin were formulated by spray-drying of a single feed aqueous solution, thus avoiding the use of organic solvents. The obtained PVA-CPx formulations were characterized by an overall smooth surface with a spherical morphology, nevertheless, the presence of a few irregular particles with external voids was detected (Figure 2 - insets). The increase of CPx content did not significantly changed particle size distribution (Figure 2), with average mean diameters ranging from $2.39 \pm 0.76 \mu m$ (PVA alone) to $2.84 \pm 0.95 \mu m$ (10 wt.\% of CPx), demonstrating the narrow size of the particles, regardless the drug content. This was also corroborated by the low coefficients of variation $(0.3-0.4)$ among the formulations, evidencing the process reproducibility and scalability. Overall, the physical diameter distributions depicted in Figure 2 were a good indicator of the suitability of these formulations for inhalation (Patton and Byron, 2007).

\subsection{Physicochemical characterization of microparticles}

The IR spectra of the PVA, spray-dried ciprofloxacin (SD-CPx) and the PVA-CPx microparticles are depicted in Figure 3a. The major absorption bands of PVA are clearly identified for the PVA-CPx formulation. The absorption band between $3550-3200 \mathrm{~cm}^{-1}$ are assigned to the $O-H$ groups. The bands at 2941 and $2912 \mathrm{~cm}^{-1}$ represent the asymmetrical and symmetrical stretching vibration of $C-H$ in the PVA backbone. Secondary in-plane $O-H$ bending and $C-H$ wagging is observed at 1415 and 1330 
$\mathrm{cm}^{-1}$. The two absorption bands at 1139 and $1087 \mathrm{~cm}^{-1}$ are due to the $C-C-C$ and $C-O$ stretching vibration, respectively (Mansur et al., 2008; Zhang et al., 2010). Ciprofloxacin characteristic absorption bands were identified in SD-CPx spectrum. The bands at 3084 and $2918 \mathrm{~cm}^{-1}$ were assigned to the $C-H$ stretching vibration from the phenyl framework; the band at $1713 \mathrm{~cm}^{-1}$ was addressed to the $-\mathrm{COOH}$ stretching vibration, whereas vibration of the phenyl framework conjugated to the carboxylic acid is at $1616 \mathrm{~cm}^{-1}$ (Dong et al., 2006; Wang et al., 2007). The stretching vibration of the $C-F$ bonds appears at $1266 \mathrm{~cm}^{-1}$. Despite most of the CPx vibration modes were overlapped by the stronger PVA absorption bands, it was possible to identify CPX characteristic bands in the PVA-CPx formulations; additionally, there were no new vibrational modes nor suppression, which indicates the lack of chemical interaction between the drug and the polymer.

The Raman spectra (Figure 3b) enabled to confirm the presence of CPx characteristic peaks. The strongest Raman bands of CPx were detected in the PVA-CPx formulation, specifically at 1383 and $1464 \mathrm{~cm}^{-1}$ attributed to $O-C-O$ symmetrical and asymmetrical stretching vibration, respectively (Yang et al., 2015). The peak at 1549 $\mathrm{cm}^{-1}$ was assigned to the stretching vibration of the quinolone ring, and the band at $1625 \mathrm{~cm}^{-1}$ was attributed to the $C=C$ asymmetrical vibration of the aromatic rings (Yang et al., 2015).

The thermal properties of the SD-CPx, PVA and the PVA-CPx microparticles were evaluated by DSC. SD-CPx showed to be stable at temperatures up to 250 으 (Figure 4). PVA and PVA-CPx microparticles presented a broad endothermic peak in the temperature range of 30 and $120 \stackrel{\circ}{ }$, assigned to the evaporation of bounded water

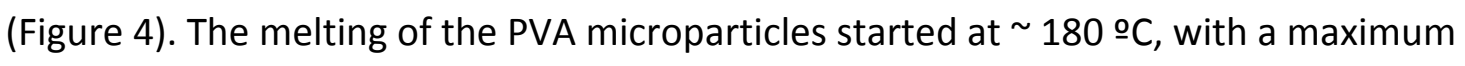


peak temperature of $220^{\circ} \mathrm{C}$. Furthermore, the polymer melting temperature remained unchanged after incorporation of the drug, suggesting that the drug and the polymer did not have a chemical affinity.

The degree of crystallinity $(\Delta X)$ of the PVA and PVA-CPx particles was calculated according to:

$$
\Delta X=\frac{\Delta H_{m}}{\Delta H_{m}^{0}}
$$

where, $\Delta H_{m}$ and $\Delta H_{m}^{0}$ are the sample melting enthalpy, and the enthalpy for $100 \%$ crystalline PVA polymer (168 J/g) (Wu et al., 2010). PVA unloaded microparticles had a crystallinity degree of $35 \%$, while the samples with 10 wt.\% CPX had a $\Delta X$ of $40 \%$, suggesting that the drug could act as a nucleation agent, leading to an increase in polymer crystallization (Wang et al., 2007).

The Hansen solubility parameters for the drug and polymer matrix is presented in Table 3. It is reported that compounds with similar values of solubility parameter, nominally $\Delta \delta<7.0 \mathrm{MPa}^{1 / 2}$, are more likely to be miscible, whereas if $\Delta \delta>10.0$ $\mathrm{MPa}^{1 / 2}$, the compounds are most probably immiscible (Greenhalgh et al., 1999). The solubility parameter of PVA is $26.34 \mathrm{MPa}^{1 / 2}$, and the CPx is $24.15 \mathrm{MPa}^{1 / 2}$ (Barton, 1990; Shah and Agrawal, 2012). The difference in the solubility parameter for the polymerdrug interaction can be estimated from:

$$
\Delta \delta_{t}=\left[\left(\delta_{P, p}-\delta_{D, p}\right)^{2}+\left(\delta_{P, h}-\delta_{D, h}\right)^{2}+\left(\delta_{P, d}-\delta_{D, d}\right)^{2}\right]^{1 / 2}
$$


where, $p, d$ and $h$ indicate the polar, dispersive and hydrogen bonding components, respectively, while the symbols $P$ and $D$ identify the polymer and drug, respectively. The difference of solubility parameter between the drug and the polymer matrix was 9.24 $\mathrm{MPa}^{1 / 2}$, which suggests that a miscibility between the polymer and the drug is unlikely to occur. The analysis of the Hansen solubility parameters corroborates the chemical (figure 4) and thermal analysis (figure 5) recorded for the polymer-drug microparticles.

The inhalable potential of the formulation containing $10 \mathrm{wt} . \%$ of CPx was further assessed by the volumetric diameter measured by laser diffraction, which is depicted in Figure 5. The volume-weighted of SD-CPx showed a median volume diameter of $7.18 \pm 0.20 \mu m$ (Figure 5 - inset) along with an irregular, crystalline-like morphology (Figure S3). On the other hand, spray-dried PVA particle size distribution was very similar to the PVA-CPx 10 wt.\% microparticles. These formulations exhibited a trimodal profile with the predominant population in the range of 1-10 $\mu \mathrm{m}$; hence, the median volume diameter of the PVA and PVA-CPx was determined as $3.30 \pm 0.03 \mu \mathrm{m}$ and $3.20 \pm 0.13 \mu \mathrm{m}$, respectively, which correlates with the diameter estimation from SEM microphotographs, and lies within the optimum inhalable size range (Healy et al., 2014). Moreover, SD-CPX particle span $\left(\operatorname{Span}=\left(D_{0.9}-D_{0.1}\right) / D_{0.5}\right)$ indicates that the SD-CPx have a wide particle size distribution, when compared to the polymeric carriers (Figure 5).

The physical stability of the formulations under a variety of humidity conditions at constant temperature of $25^{\circ} \mathrm{C}$ was evaluated by dynamic vapor sorption (DVS). The moisture sorption-desorption profiles (Figure 6) of SD-CPx, PVA and PVA-CPx showed a maximum mass change of $1.9 \%, 25 \%$ and $23 \%$, respectively, at a $90 \% \mathrm{RH}$. All 
formulations exhibited reversible isotherms with only minimal hysteresis, which indicates that despite being moisture sensitive, this effect is reversible. These results agree with a previous work using PVA-drug particles (Salama et al., 2008a), which reports a water absorption profile up to $90 \% \mathrm{RH}$, where a maximum mass change about $25 \%$ for the formulation containing 90 wt.\% PVA was reported.

\subsection{In vitro studies: CPx loading and drug release}

The PVA-CPx microparticles were produced in a single -step by spray-drying of an environmentally friendly aqueous solution with high encapsulation efficiencies (>90\%, Table 4). The increase of CPx up to 10 wt.\% related to the PVA mass did not affect the drug entrapment, suggesting the potential to further tailor CPx loading of PVA formulations, according to the clinical requirements.

There is no standard in vitro methodology for assessing drug release profile from polymeric inhaled powders that mimics the lung environment (Salama et al., 2008b); however, the dialysis membrane method has been extensively used and its regarded as a suitable set up to assess drug dissolution in the lungs (Tewes et al., 2016). The release profiles over time for the different formulations at physiologic conditions $\left(\mathrm{pH} 7.4\right.$, and $\left.37^{\circ} \mathrm{C}\right)$ are shown in Figure 7. SD-CPx presented a burst release (90\%) during the first hour, while PVA formulations showed a sustained release of ciprofloxacin over 24h. Formulations with lower CPx contents ( 2 and 5 wt.\%) presented similar release profiles, while the 10 wt.\% formulation showed a rapid initial release after 10 minutes $(40.1 \pm 7.0 \%)$, representing approximately a 4-fold increase comparing with the lowest $\mathrm{CPx}$ formulations $(8.0 \pm 0.4 \%$ and $12.1 \pm 2.5 \%)$. The increasing of CPx content probably lead to spatial constraints and to an increase of the 
CPx molecules closer to the microparticle boundaries, thus reducing the diffusion path of the drug, boosting its initial release.

The experimental data was modelled using the Korsmeyer-Peppas model for the first hour, where $M_{t} / M_{\infty}<0.6$ (Bruschi, 2015), and the kinetic parameters are presented in Table 5. The estimation of the parameter $k$ corroborates that higher CPx content increases the release rate when comparing with lower formulations $(p<0.001)$. The values of the release exponent $n$ for the different formulations also reflects distinct release mechanisms. For a spherical geometry, $n<0.43$ is indicative of Fickian diffusion, whereas a superimposition of drug diffusion and polymer chain relaxation is described by $0.43<n<0.85$ (Ritger and Peppas, 1987a; Siepmann and Peppas, 2001). Our results suggest that the increase in the CPx content leads to a primarily diffusion-dependent release mechanism, while for the lower CPx formulation the release mechanism relies on the superimposition of both phenomena (drug diffusion and polymer chain relaxation).

However, these results are merely indicative of the microparticles performance in the PBS. For instance, a study by Arora et al. (Arora et al., 2015) using dry powder formulations of voriconazole, reports differences in the kinetic parameters upon different in vitro methodologies, which are related to the water content in the system.

\subsection{In vitro aerodynamic performance}

Aerodynamic performance of the spray-dried formulations was tested using the NGI for the assessment of the lung deposition pattern of the drug. The particle deposition on each stage of the NGI is plotted in Figure 8. For both formulations, SD-CPx and PVA$\mathrm{CPx}$, the delivered dose was $72.16 \pm 1.76 \%$ and $84.08 \pm 1.31 \%$ of the metered 
dose, respectively. Some degree of particle aggregation may have occurred since a substantial fraction of the powder was deposited in the throat and in the upper NGI stages, which also explains the increased aerodynamic median diameter compared with geometric estimation. Nevertheless, PVA-CPx deposition showed a significant decrease in the device and throat when compared with the SD-CPx, and an increase in the lower stages of the NGI. Better aerodynamic features were also obtained for the PVA-based formulation rather than SD-CPx; despite presenting similar MMAD and GSD values, the determined FPF $(<5 \mu m)$ for PVA-CPx $(39.78 \pm 0.98 \%)$ was statistically higher $(p<0.01)$ than SD-CPx $(30.43 \pm 1.38 \%)$, meaning that this fraction can reach the deep lungs. The obtained parameters are above the range of conventional dry powder inhaler formulations (15-30 \%) (Saigal et al., 2013; Smith and Parry-Billings, 2003).

Other authors have reported the production of inhalable PVA-based formulations with different FPF values. Salama and co-workers (Salama et al., 2008a) described a linear relationship between PVA content and FPF, achieving $30.76 \pm 1.5 \%$ for a PVA content of $90 \%(w / w)$. Other PVA-based systems for the encapsulation of nifedipine by spraydrying, presented MMAD between 8.05 and $>13 \mu \mathrm{m}$ and a FPF between $20.4 \pm 2.47$ and $34.9 \pm 2.4 \%$ (Saigal et al., 2013).

\section{Conclusion}

This study provides an insight in the processing and performance of inhalable spraydried formulations of PVA for the delivery of ciprofloxacin. An orthogonal design was used to assess the effect of the processing parameters in the particle's distribution 
diameter. The order of importance of each factor was determined as polymer concentration $>$ feed rate $>$ inlet temperature.

The incorporation of ciprofloxacin up to $10 \mathrm{wt} . \%$ was achieved under the optimal processing parameters, with efficiencies higher than $90 \%$, using a simple and scalable process with in vitro profiles showing a sustained release over $24 \mathrm{~h}$. Moreover, preliminary aerosolization studies using a NGI confirmed the suitability of the formulation to be administrated as a DPI, showing a FPF $(<5 \mu m)$ of $39.78 \pm 0.98 \%$. Overall, this study describes a promising platform for the pharmaceutical tailoring of DPIs using a synthetic, biodegradable and GRAS (generally regarded as safe) polymer.

\section{Acknowledgments}

The authors acknowledge Dr. Patricia Hayes for the help with the Raman experiments. This research did not receive any specific grant from funding agencies in the public, commercial, or not-for-profit sectors.

\section{References}

Adi, H., Young, P.M., Chan, H.-K., Salama, R., Traini, D., 2010. Controlled release antibiotics for dry powder lung delivery. Drug Development and Industrial Pharmacy $36,119-126$.

Arora, S., Haghi, M., Loo, C.-Y., Traini, D., Young, P.M., Jain, S., 2015. Development of an Inhaled Controlled Release Voriconazole Dry Powder Formulation for the Treatment of Respiratory Fungal Infection. Molecular Pharmaceutics 12, 2001-2009.

Atkins, P.J., 2005. Dry Powder Inhalers: An Overview. Respiratory Care 50, 1304-1312. 
Barton, A.F.M., 1990. Handbook of Polymer-Liquid Interaction Parameters and Solubility Parameters. Taylor \& Francis.

Bruschi, M.L., 2015. 5 - Mathematical models of drug release Strategies to Modify the Drug Release from Pharmaceutical Systems. Woodhead Publishing, pp. 63-86.

Chaouat, M., Le Visage, C., Baille, W.E., Escoubet, B., Chaubet, F., Mateescu, M.A., Letourneur, D., 2008. A Novel Cross-linked Poly(vinyl alcohol) (PVA) for Vascular Grafts. Advanced Functional Materials 18, 2855-2861.

Dailey, L.A., Jekel, N., Fink, L., Gessler, T., Schmehl, T., Wittmar, M., Kissel, T., Seeger, W., 2006. Investigation of the proinflammatory potential of biodegradable nanoparticle drug delivery systems in the lung. Toxicology and Applied Pharmacology $215,100-108$.

Dong, Z., Wang, Q., Du, Y., 2006. Alginate/gelatin blend films and their properties for drug controlled release. Journal of Membrane Science 280, 37-44.

Farr, S.J., Otulana, B.A., 2006. Pulmonary delivery of opioids as pain therapeutics. Advanced Drug Delivery Reviews 58, 1076-1088.

Felice, B., Prabhakaran, M.P., Zamani, M., Rodríguez, A.P., Ramakrishna, S., 2015. Electrosprayed poly(vinyl alcohol) particles: preparation and evaluation of their drug release profile. Polymer International 64, 1722-1732.

Greenhalgh, D.J., Williams, A.C., Timmins, P., York, P., 1999. Solubility parameters as predictors of miscibility in solid dispersions. Journal of Pharmaceutical Sciences 88, 1182-1190. 
Hara, K., Tsujimoto, H., Tsukada, Y., Huang, C.C., Kawashima, Y., Tsutsumi, M., 2008. Histological examination of PLGA nanospheres for intratracheal drug administration. International Journal of Pharmaceutics 356, 267-273.

Healy, A.M., Amaro, M.I., Paluch, K.J., Tajber, L., 2014. Dry powders for oral inhalation free of lactose carrier particles. Advanced Drug Delivery Reviews 75, 32-52.

Labiris, N.R., Dolovich, M.B., 2003. Pulmonary drug delivery. Part I: Physiological factors affecting therapeutic effectiveness of aerosolized medications. British Journal of Clinical Pharmacology 56, 588-599.

Lee, Y., Filliben, J.J., Micheals, R.J., Jonathon Phillips, P., 2013. Sensitivity analysis for biometric systems: A methodology based on orthogonal experiment designs. Computer Vision and Image Understanding 117, 532-550.

Manca, M.L., Peris, J.E., Melis, V., Valenti, D., Cardia, M.C., Lattuada, D., EscribanoFerrer, E., Fadda, A.M., Manconi, M., 2015. Nanoincorporation of curcumin in polymerglycerosomes and evaluation of their in vitro-in vivo suitability as pulmonary delivery systems. RSC Adv. 5, 105149-105159.

Manca, M.L., Roberta, C., Donatella, V., Sonia, T., Teresa, F., Nevio, P., M., F.A., Maria, M., 2013. Isoniazid-gelatin conjugate microparticles containing rifampicin for the treatment of tuberculosis. Journal of Pharmacy and Pharmacology 65, 1302-1311.

Manca, M.L., Valenti, D., Sales, O.D., Nacher, A., Fadda, A.M., Manconi, M., 2014. Fabrication of polyelectrolyte multilayered vesicles as inhalable dry powder for lung administration of rifampicin. International Journal of Pharmaceutics 472, 102-109. 
Mansur, H.S., Sadahira, C.M., Souza, A.N., Mansur, A.A.P., 2008. FTIR spectroscopy characterization of poly (vinyl alcohol) hydrogel with different hydrolysis degree and chemically crosslinked with glutaraldehyde. Materials Science and Engineering: C 28, 539-548.

Patton, J.S., 1996. Mechanisms of macromolecule absorption by the lungs. Advanced Drug Delivery Reviews 19, 3-36.

Patton, J.S., Byron, P.R., 2007. Inhaling medicines: delivering drugs to the body through the lungs. Nat Rev Drug Discov 6, 67-74.

Pilcer, G., Amighi, K., 2010. Formulation strategy and use of excipients in pulmonary drug delivery. International Journal of Pharmaceutics 392, 1-19.

Rawat, A., Majumder, Q.H., Ahsan, F., 2008. Inhalable large porous microspheres of low molecular weight heparin: In vitro and in vivo evaluation. Journal of Controlled Release 128, 224-232.

RenJie, L., 2008. Orthogonal test design for optimization of the extraction of polysaccharides from Phascolosoma esulenta and evaluation of its immunity activity. Carbohydrate Polymers 73, 558-563.

Ritger, P.L., Peppas, N.A., 1987a. A simple equation for description of solute release I. Fickian and non-fickian release from non-swellable devices in the form of slabs, spheres, cylinders or discs. Journal of Controlled Release 5, 23-36. 
Ritger, P.L., Peppas, N.A., 1987b. A simple equation for description of solute release II. Fickian and anomalous release from swellable devices. Journal of Controlled Release 5, $37-42$.

Rybak, M.J., Abate, B.J., Kang, S.L., Ruffing, M.J., Lerner, S.A., Drusano, G.L., 1999. Prospective Evaluation of the Effect of an Aminoglycoside Dosing Regimen on Rates of Observed Nephrotoxicity and Ototoxicity. Antimicrobial Agents and Chemotherapy 43, 1549-1555.

Saigal, A., Ng, W.K., Tan, R.B.H., Chan, S.Y., 2013. Development of controlled release inhalable polymeric microspheres for treatment of pulmonary hypertension. International Journal of Pharmaceutics 450, 114-122.

Sakagami, M., Byron, P.R., 2005. Respirable Microspheres for Inhalation. Clinical Pharmacokinetics 44, 263-277.

Salama, R., Hoe, S., Chan, H.-K., Traini, D., Young, P.M., 2008a. Preparation and characterisation of controlled release co-spray dried drug-polymer microparticles for inhalation 1: Influence of polymer concentration on physical and in vitro characteristics. European Journal of Pharmaceutics and Biopharmaceutics 69, 486-495.

Salama, R.O., Traini, D., Chan, H.-K., Young, P.M., 2008b. Preparation and characterisation of controlled release co-spray dried drug-polymer microparticles for inhalation 2: Evaluation of in vitro release profiling methodologies for controlled release respiratory aerosols. European Journal of Pharmaceutics and Biopharmaceutics 70, 145-152. 
Schneider, C.A., Rasband, W.S., Eliceiri, K.W., 2012. NIH Image to ImageJ: 25 years of image analysis. Nat Meth 9, 671-675.

Shah, M., Agrawal, Y., 2012. Ciprofloxacin hydrochloride-loaded glyceryl monostearate nanoparticle: factorial design of Lutrol F68 and Phospholipon 90G. Journal of Microencapsulation 29, 331-343.

Siepmann, J., Peppas, N.A., 2001. Modeling of drug release from delivery systems based on hydroxypropyl methylcellulose (HPMC). Advanced Drug Delivery Reviews 48, 139-157.

Sivadas, N., O’Rourke, D., Tobin, A., Buckley, V., Ramtoola, Z., Kelly, J.G., Hickey, A.J., Cryan, S.-A., 2008. A comparative study of a range of polymeric microspheres as potential carriers for the inhalation of proteins. International Journal of Pharmaceutics $358,159-167$.

Smith, I.J., Parry-Billings, M., 2003. The inhalers of the future? A review of dry powder devices on the market today. Pulmonary Pharmacology \& Therapeutics 16, 79-95.

Tewes, F., Brillault, J., Lamy, B., O'Connell, P., Olivier, J.-C., Couet, W., Healy, A.M., 2016. Ciprofloxacin-Loaded Inorganic-Organic Composite Microparticles To Treat Bacterial Lung Infection. Molecular Pharmaceutics 13, 100-112.

Trapani, A., Di Gioia, S., Ditaranto, N., Cioffi, N., Goycoolea, F.M., Carbone, A., GarciaFuentes, M., Conese, M., Alonso, M.J., 2013. Systemic heparin delivery by the pulmonary route using chitosan and glycol chitosan nanoparticles. International Journal of Pharmaceutics 447, 115-123. 
Ungaro, F., d'Angelo, I., Coletta, C., d'Emmanuele di Villa Bianca, R., Sorrentino, R., Perfetto, B., Tufano, M.A., Miro, A., La Rotonda, M.I., Quaglia, F., 2012. Dry powders based on PLGA nanoparticles for pulmonary delivery of antibiotics: Modulation of encapsulation efficiency, release rate and lung deposition pattern by hydrophilic polymers. Journal of Controlled Release 157, 149-159.

Varanda, F., Pratas de Melo, M.J., Caço, A.I., Dohrn, R., Makrydaki, F.A., Voutsas, E., Tassios, D., Marrucho, I.M., 2006. Solubility of Antibiotics in Different Solvents. 1. Hydrochloride Forms of Tetracycline, Moxifloxacin, and Ciprofloxacin. Industrial \& Engineering Chemistry Research 45, 6368-6374.

Vild, A., Teixeira, S., Kühn, K., Cuniberti, G., Sencadas, V., 2016. Orthogonal experimental design of titanium dioxide-Poly(methyl methacrylate) electrospun nanocomposite membranes for photocatalytic applications. Journal of Environmental Chemical Engineering 4, 3151-3158.

Wang, Q., Dong, Z., Du, Y., Kennedy, J.F., 2007. Controlled release of ciprofloxacin hydrochloride from chitosan/polyethylene glycol blend films. Carbohydrate Polymers 69, 336-343.

Woods, A., Patel, A., Spina, D., Riffo-Vasquez, Y., Babin-Morgan, A., de Rosales, R.T.M., Sunassee, K., Clark, S., Collins, H., Bruce, K., Dailey, L.A., Forbes, B., 2015. In vivo biocompatibility, clearance, and biodistribution of albumin vehicles for pulmonary drug delivery. Journal of Controlled Release 210, 1-9.

Wu, Q., Chen, N., Wang, Q., 2010. Crystallization behavior of melt-spun poly(vinyl alcohol) fibers during drawing process. Journal of Polymer Research 17, 903-909. 
Xie, G., Chen, Z., Ramakrishna, S., Liu, Y., 2015. Orthogonal design preparation of phenolic fiber by melt electrospinning. Journal of Applied Polymer Science 132, n/an/a.

Yang, L., Qin, X., Jiang, X., Gong, M., Yin, D., Zhang, Y., Zhao, B., 2015. SERS investigation of ciprofloxacin drug molecules on TiO2 nanoparticles. Physical Chemistry Chemical Physics 17, 17809-17815.

Zahoor, A., Sharma, S., Khuller, G.K., 2005. Inhalable alginate nanoparticles as antitubercular drug carriers against experimental tuberculosis. International Journal of Antimicrobial Agents 26, 298-303.

Zhang, Y., Zhu, P.C., Edgren, D., 2010. Crosslinking reaction of poly(vinyl alcohol) with glyoxal. Journal of Polymer Research 17, 725-730.

Zheng, P., Liu, H., Wang, J., Yu, B., Zhang, B., Yang, R., Wang, X., 2014. Optimization of experimental conditions by orthogonal test design in a laser-induced breakdown experiment to analyze aluminum alloys. Analytical Methods 6, 2163-2169. 


\section{Figure Captions}

Figure 1 - Orthogonal design: (a) dependence of average particle diameter and coefficient of variation (Cv) on the processing factors and levels by spray-drying, determined by measurements using Image J software; (b) viscosity of PVA feed solutions $(0.5 \%, 1 \%$ and $2 \% \mathrm{w} / \mathrm{v})$ as a function of the shear rate measured at $25 \% \mathrm{C}(\mathrm{b})$.

Figure 2 - Particle size distribution of PVA with different CPx contents $(0,2,5$ and 10 wt.\%). Insets: representative electron micrographs of (a) PVA and (b) PVA-CPx 10 wt.\% microparticles (scale bar: $5 \mu \mathrm{m}$ ).

Figure 3 - Fourier transformed infrared spectra (a) and Raman spectra (b) of the spraydried formulations.

Figure 4 - DSC of spray-dried ciprofloxacin and PVA formulations.

Figure 5 - Volume-weighted particle size distribution and geometric parameters (inset) of SD-CPx, PVA and PVA-CPx 10wt.\% formulations measured by laser diffraction.

Figure 6 - Dynamic vapor sorption isotherms $(a-c)$ and mass change versus time $(d-f)$ for SD-CPx, PVA and PVA-CPx 10wt.\% formulations. 
Figure 7 - Ciprofloxacin release profiles from free SD-CPx and PVA-CPx formulations using dialysis membrane method in PBS $\left(\mathrm{pH} \mathrm{7.4)}\right.$ at $37 \pm 0.5^{\circ} \mathrm{C}$. Values are presented as average $\pm S D$ of 3 measurements.

Figure 8 - Aerodynamic particle size distribution obtained by NGI at a flow rate of 60 L/min. Inset: Aerodynamic parameters for the PVA-CPx spray-dried formulation compared with spray-dried CPx as a control. Results are presented as mean \pm SD ( $n=3 ; * p<0.05, * * p<0.01, * * * p<0.001)$. 
https://doi.org/10.1016/j.ijpharm.2018.05.060

\section{Graphical Abstract}

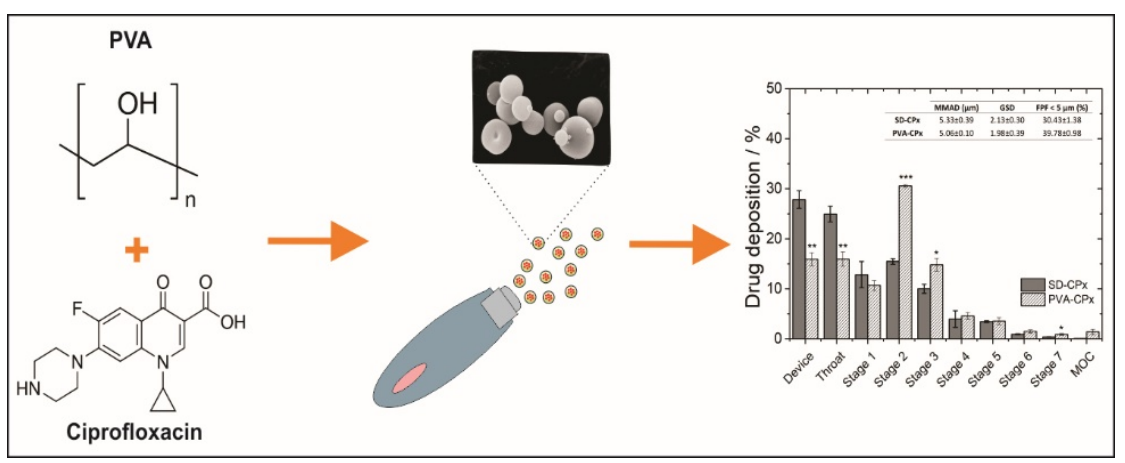



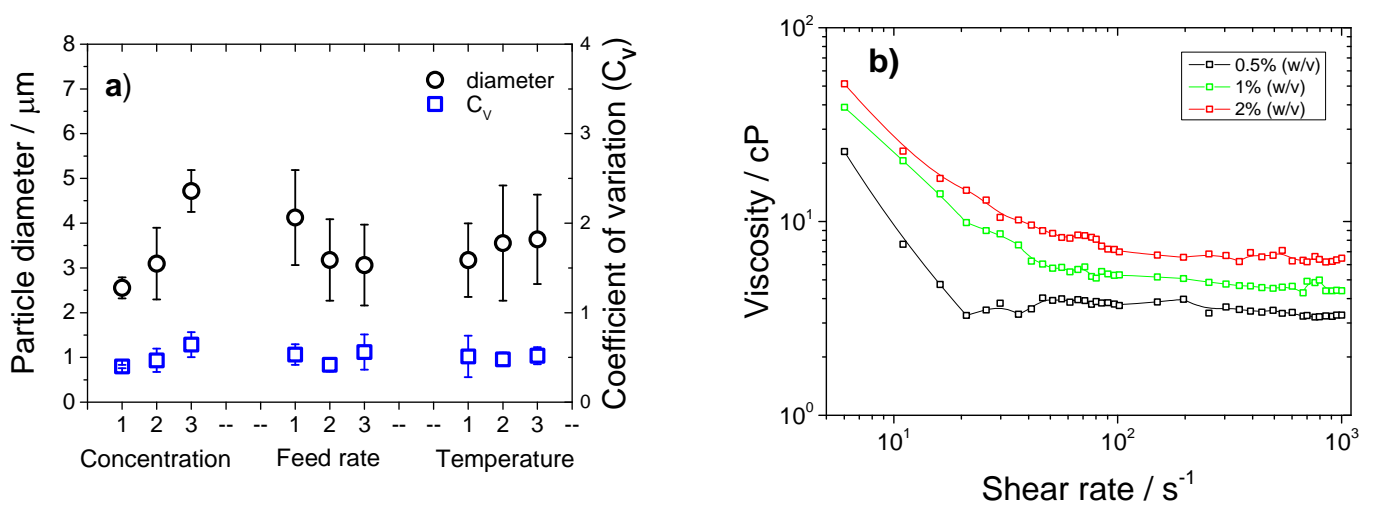

Figure 1 


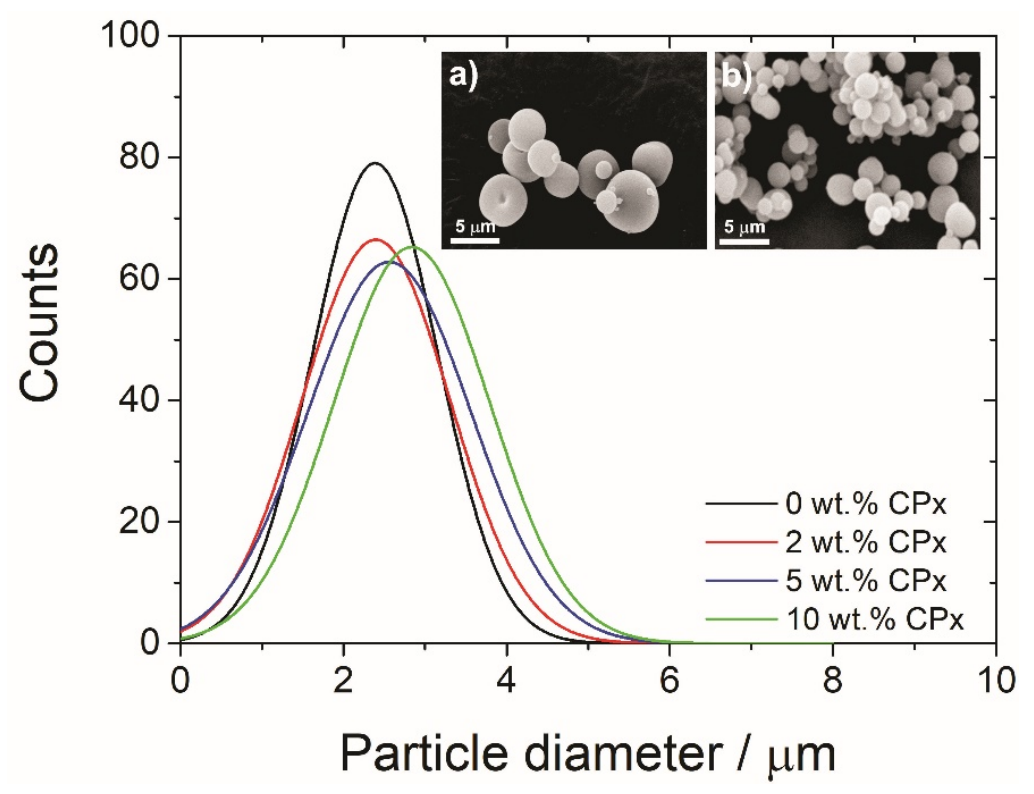

Figure 2 

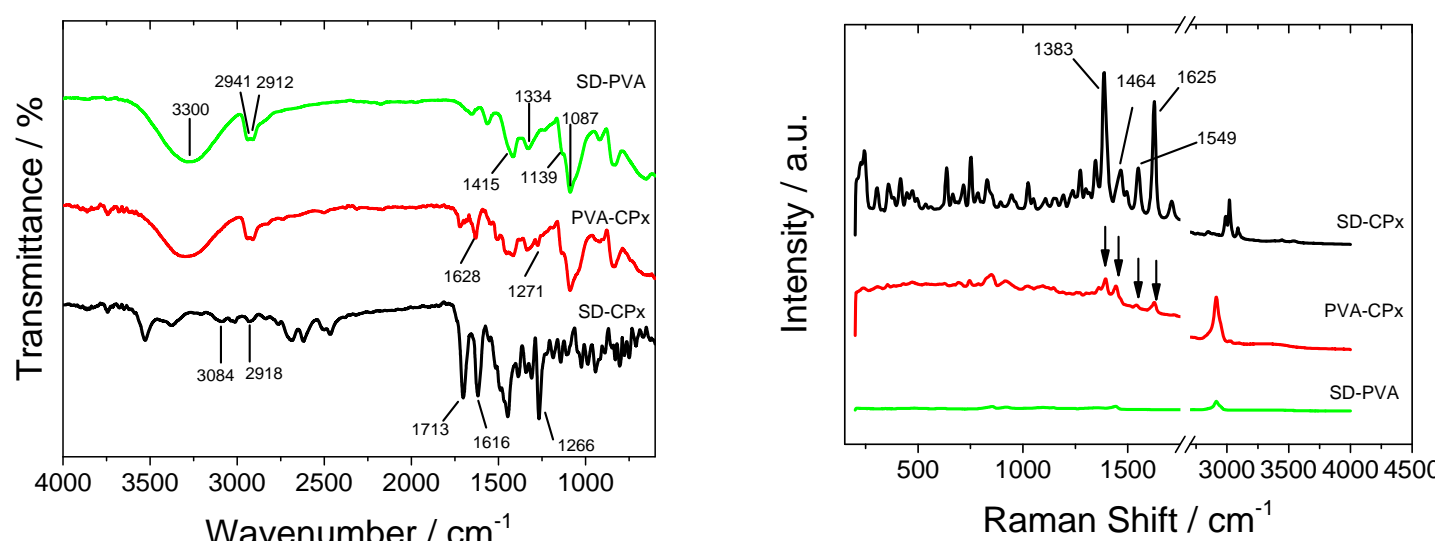

Figure 3 


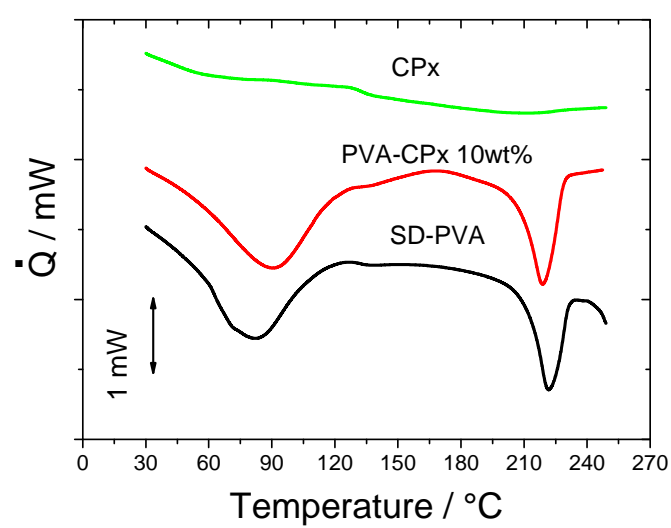

Figure 4 


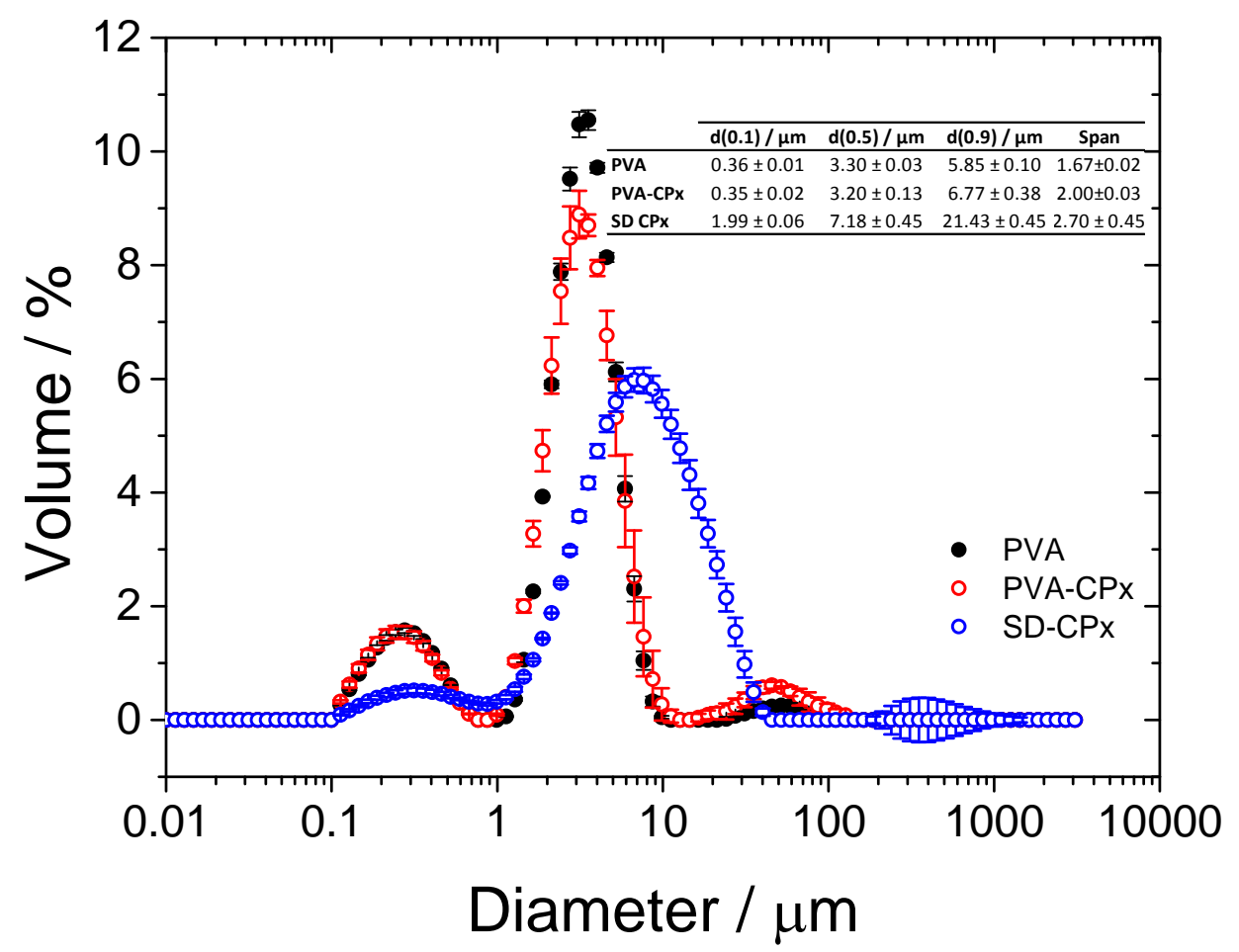

Figure 5 

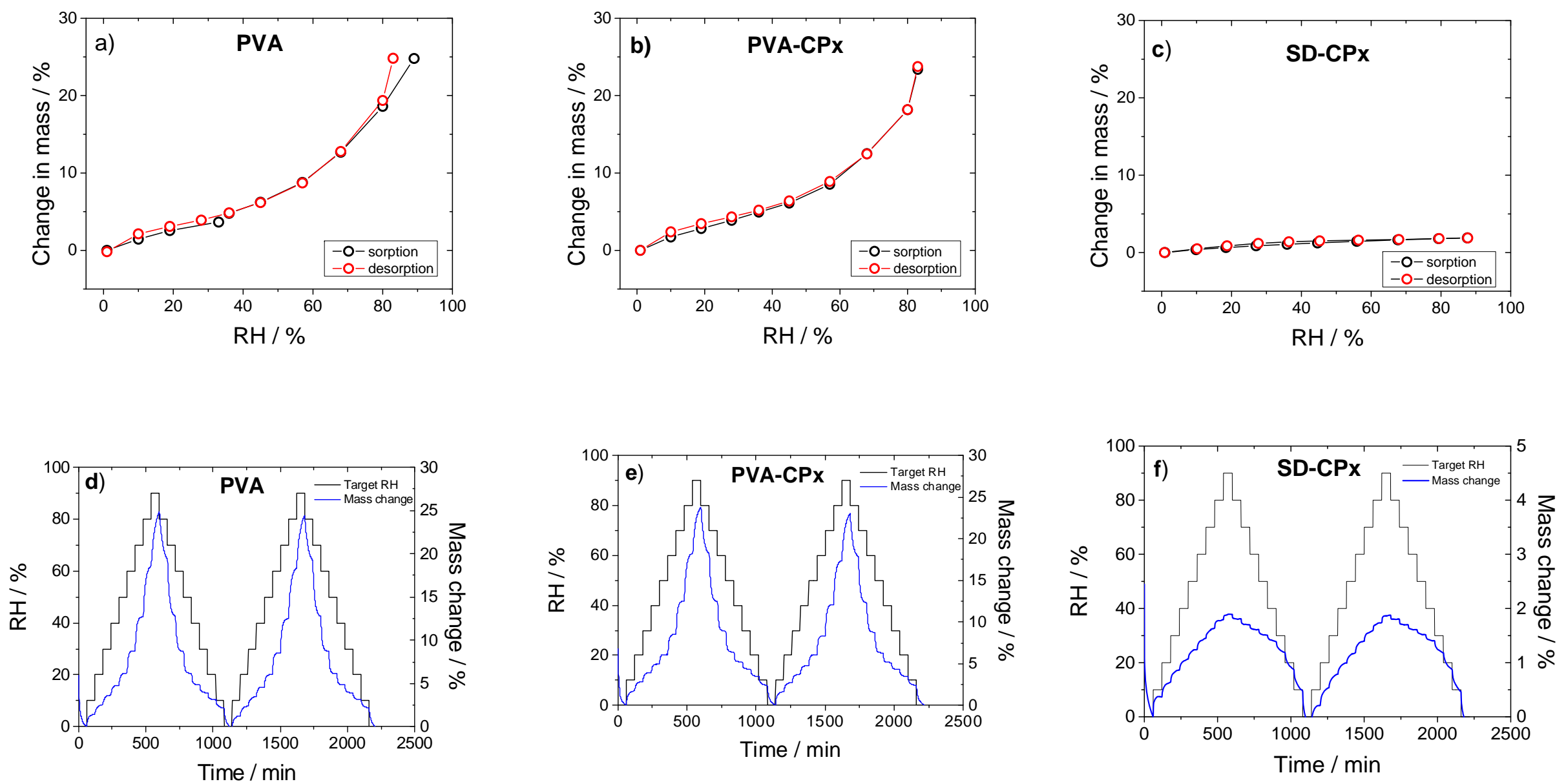

\section{Figure 6}


https://doi.org/10.1016/j.ijpharm.2018.05.060 


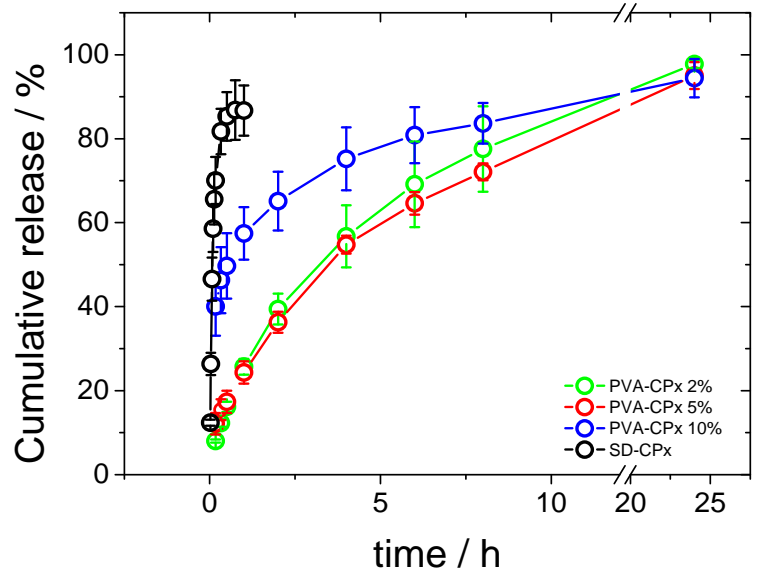

Figure 7 


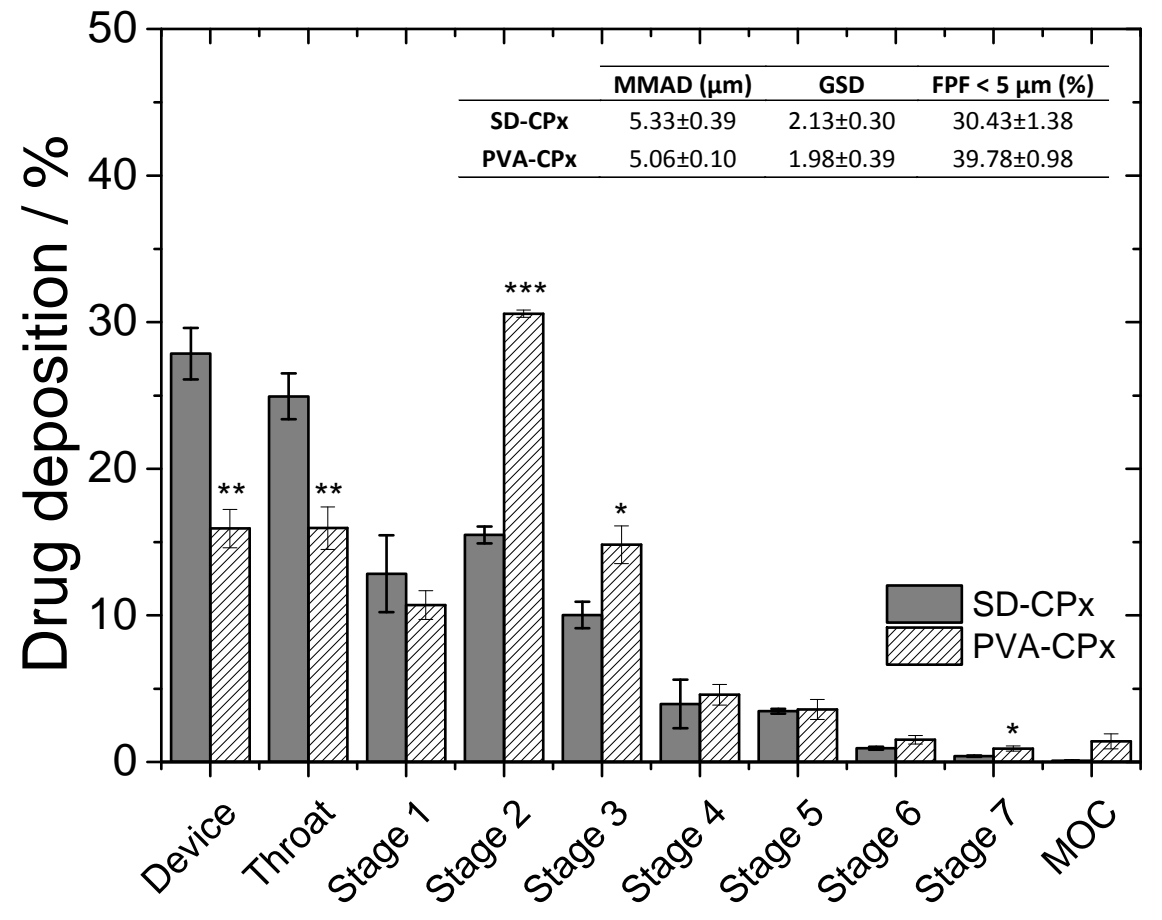

Figure 8 